\title{
A New Image Segmentation Algorithm Based on Particle Swarm Optimization and Rough Set
}

\author{
Wafaa Ghonaim ${ }^{1 *}$, Shereen A. Taie ${ }^{2}$ \\ ${ }^{1}$ Faculty of Science, Al-Azhar University, Cairo, Egypt. \\ 2 Computer Science Department, Faculty of Computers and Information, Fayoum University, Egypt. \\ * Corresponding author. Email: dr.wafaaghonaim@yahoo.com \\ Manuscript submitted July 28, 2016; accepted January 7, 2017 \\ doi: $10.17706 /$ jcp.13.1.130-138
}

\begin{abstract}
Image segmentation is a basic technique for advanced image analysis. In this paper a new image segmentation algorithm based on combining particle swarm optimization PSO and rough set is proposed. The algorithm adopts mean roughness measure as evaluation standard, this measure depends on minimization of roughness in both object and background regions; by determining the optimal threshold of partitioning. In this algorithm, threshold estimation is regarded as a search procedure that searches for an optimal value in a continuous gray-scale interval. The results of the PSO based proposed algorithm are compared with Bat-Inspired algorithm under mean roughness measure as the fitness function and simulations show that the PSO seems much superior than Bat algorithm.
\end{abstract}

Key words: Particle swarm optimization, bat-inspired algorithm, rough set, image segmentation, mean roughness measure.

\section{Introduction}

Image segmentation refers to the process of partitioning a digital image to related homogenous sections or regions, where the homogeneity of a region may be composed based on different criteria such as gray level. The goal of segmentation is to simplify the representation of an image into different disjoint regions that are more meaningful and easier to analyze [1], [2].

Segmentation algorithms are based on two significant criteria: the homogeneity of a region (thresholding) and the discontinuity between adjacent disjoint regions (finding edges). Since the segmented image obtained from the homogeneity criterion has the advantage of smaller storage space, fast processing speed and easy in manipulation, thresholding techniques are considered the most popular. Thresholding techniques can be classified into two categories: bi-level and multi-level. In bi-level, one limit value is chosen to segment an image into two classes: one representing the object and the other one segmenting the background. When distinct objects are depicted within a given scene, multiple threshold values have to be selected for proper segmentation, which is commonly called multi-level thresholding [3], [4].

Image segmentation has received a lot of attention by the research people and a variety of image segmentation schemes have been proposed. In [5], a fast Synthetic Aperture Radar (SAR) image segmentation method based on Artificial Bee Colony ( $A B C$ ) algorithm is proposed, the method regards threshold estimation as a search process and employs $\mathrm{ABC}$ algorithm to optimize it, experimental results indicate that the segmentation quality of the method is benefit from the improved two-dimensional grey entropy, for the fact that noise almost completely disappears, and most useful information about edge and 
texture is preserved. An overview of PSO based approaches in image segmentation is provided in [3], and illustrated that PSO itself is a very powerful technique, but when combined with other computational intelligence techniques results in a more effective approach.

Segmentation in gray scale images results in ill-defined object boundaries [6]. Due to the formidable ability processing information uncertainty, the rough set theory has become a new academic hot spot in the artificial intelligence domain, In machine learning, knowledge acquisition, decision analysis, process control, image processing and many other fields, it has been widely used [7].

In this paper, PSO combined with rough set based on the mean roughness measure as the evaluation standard and use PSO to segment image in order to obtain better results. The advantages of PSO are that, easy to implement and there are few parameters to adjust. PSO has been successfully applied in many areas: function optimization, artificial neural network training, fuzzy system control, and other areas [2]. The rest of this paper is arranged as follows. Section 2, introduces an overview of the particle swarm optimization. Section 3, gives an overview of rough set theory. Section 4 presented the algorithm description. Experimental results are discussed in Section 5. Section 6, shows the comparative results of applying Bat algorithm. Finally, Conclusion is presented in Section 7.

\section{Particle Swarm Optimization}

Optimization is the act of obtaining the best result under given circumstances. PSO is an evolutionary optimization technique introduced by Kennedy and Eberhart at 1995. It has been widely used to solve a wide range of optimization problems. The PSO concept is based on social behavior of bird flocking. In the PSO algorithm, the particles move in the multi-dimensional search space [8], [9]. PSO is a population-based optimization technique that exploits a population of individuals to search promising regions of the function space. The population is called swarm and the individuals are called particles, each particle is a solution to the problem and moves through the search space with an adaptable velocity according to a certain rule [10]. Each particle has fitness value which is calculated by using fitness function and searches the point that minimizes the fitness function. In PSO particle's positions and velocities are generated randomly then the velocity and position of a particle are updated at t time according to the following rules represented by equation (1) and (2) respectively.

$$
\begin{gathered}
X_{i d}(t+1)=X_{i d}(t)+V_{i d}(t+1) \\
V_{i d}(t+1)=w \times V_{i d}(t)+C_{1} r_{1}\left(\text { Pbest }_{i d}-X_{i d}(t)\right)+C_{2} r_{2}\left(\text { gbest }_{d}-X_{i d}(t)\right),
\end{gathered}
$$

where $-V_{\min }<V_{i d} \leq V_{\max }$, and $\quad i=1,2, \ldots \ldots . p s$. Where $p s$ denotes the population size, $\mathrm{w}$ is the inertia weight which has the effect of improving the convergence of search by reducing the speed as time progresses. Pbest is the best previous solution of the $i^{\text {th }}$ particle that is recorded, gbest is the best particle among the whole population, and constants $C_{1}$ and $C_{2}$ are weighting factors, which pull each particle towards pbest and gbest positions. Where $r_{1}$ and $r_{2}$ are two random values between 0 and 1 [11].

\section{Rough Set Theory}

Rough set theory was developed by Zdzislaw Pawlak in the early eighties. It belongs to the family of concepts and mathematical approaches; the rough set theory is a convenient and powerful tool in theoretical and practical researches in the field of representing incomplete knowledge and incompletely defined sets. It deals with the classificatory analysis of data tables. The data may be acquired from measurements or from human experts'. The purpose of developing rough set is motivated by the practical needs in classification and concept formation with incomplete information [12], [13]. The main goal of the 
rough set analysis is to synthesis approximation of concepts from the acquired data. That is rough set theory can be perceived as a new mathematical tool to deal with vagueness and uncertainty [14].

Let the universe $U$ be an image of size $\left(m_{\mathrm{I}} \times n_{\mathrm{I}}\right)$ consisting of a collection of pixels, if we partition $U$ into a collection of non-overlapping $M$ blocks, each block of size $m \times n$, say. Let us consider an object-background separation a two class problem of an $m_{I} \times n_{\mathrm{l}}, L$ gray level, with gray level intervals $(0,1, \ldots \ldots T$ and $T+1$, $T+2, \ldots \ldots, L-1$ ); Let $B$ and prop $O$ represent two properties that characterize background and object regions, respectively. Object and background can be viewed as two sets with their rough representation with respect to gray level $T$ as defined in [6] is given as follows:-

Lower approximations of the object $\left(\underline{\mathrm{O}}_{\mathrm{T}}\right)$ :

$$
\underline{\mathrm{O}}_{\mathrm{T}}=\left\{\bigcup_{\mathrm{i}} B_{\text {Block }} / P_{j}>T, \forall j=1 \ldots m \times n\right\}
$$

Upper approximation of the object $\left(\overline{\mathrm{O}}_{\mathrm{T}}\right)$ :

$$
\overline{\mathrm{O}}_{\mathrm{T}}=\left\{\bigcup_{\mathrm{i}} \text { Block }_{i}, \exists j, j=1, \ldots, \operatorname{mxn} \text { s.t. } P_{j}>T\right\},
$$

Lower approximations of the background $\left(\underline{B}_{T}\right)$ :

$$
\underline{\mathrm{B}}_{\mathrm{T}}=\left\{\bigcup_{\mathrm{i}} \text { Block }_{i} / P_{j} \leq T, \forall j=1, \ldots, m \times n\right\},
$$

Upper approximations of the background $\left(\overline{\mathrm{B}}_{\mathrm{T}}\right)$ :

$$
\overline{\mathrm{B}}_{\mathrm{T}}=\left\{\bigcup_{\mathrm{i}} \text { Block }_{i}, \exists j, j=1, \ldots, m x \text {, s.t. } P_{j} \leq T\right\},
$$

where $P_{j}$ is a pixel belong to Block. In [11], Pawlak defines the roughness of object $\mathrm{R}_{\mathrm{O}_{\mathrm{T}}}$ and background $\mathrm{R}_{\mathrm{B}_{\mathrm{T}}}$ as in equation (7) and (8) respectively.

$$
\begin{aligned}
& \mathrm{R}_{\mathrm{O}_{\mathrm{T}}}=1-\frac{\left|\underline{\mathrm{O}}_{\mathrm{T}}\right|}{\left|\overline{\mathrm{O}}_{\mathrm{T}}\right|}=\frac{\left|\overline{\mathrm{O}}_{\mathrm{T}}\right|-\left|\underline{\mathrm{O}}_{\mathrm{T}}\right|}{\left|\overline{\mathrm{O}}_{\mathrm{T}}\right|} \\
& \mathrm{R}_{\mathrm{B}_{\mathrm{T}}}=1-\frac{\left|\underline{\mathrm{B}}_{\mathrm{T}}\right|}{\left|\overline{\mathrm{B}}_{\mathrm{T}}\right|}=\frac{\left|\overline{\mathrm{B}}_{\mathrm{T}}\right|-\left|\underline{\mathrm{B}}_{\mathrm{T}}\right|}{\left|\overline{\mathrm{B}}_{\mathrm{T}}\right|}
\end{aligned}
$$

where $\left|\underline{\mathrm{O}}_{\mathrm{T}}\right|$ and $\left|\overline{\mathrm{O}}_{\mathrm{T}}\right|$ are the cardinality of the sets $\underline{\mathrm{O}}_{\mathrm{T}}$ and $\overline{\mathrm{O}}_{\mathrm{T}}$ respectively. Where $\left|\underline{\mathrm{B}}_{\mathrm{T}}\right|$ and $\left|\overline{\mathrm{B}}_{\mathrm{T}}\right|$ are the cardinality of the sets $\underline{B}_{T}$ and $\overline{\mathrm{B}}_{\mathrm{T}}$.

In [15], roughness measure for an image is denotes as the average of roughness of object and background at a certain threshold $\mathrm{T}$ and defined in equation (9),

$$
R M_{T}=\frac{\mathrm{R}_{\mathrm{O}_{\mathrm{T}}}+\mathrm{R}_{\mathrm{B}_{\mathrm{T}}}}{2}
$$

The value of $R M_{T}$ determines the roughness of the region determined and lies between 0 and 1.

\section{Algorithm Description}

In this paper an image segmentation algorithm based on combining particle swarm optimization and rough set is proposed. First of all, the algorithm is initialized with a group of random particles (threshold $T$ ), input image divided under $m \times n$ block size, and then find out the maximum gray value and the minimum value of the entire image pixels, calculate the lower approximation, upper approximation of the object and background then calculate the roughness of object and background. After find out the roughness of object 
and background calculate roughness measure. Throughout the process, each particle $i$ monitors three values its current position $X_{i d}$ the best position it reached in previous cycles pbest $_{i d}$; it's flying velocity $V_{i d}$. In each run (cycle), the position gbest ${ }_{\mathrm{d}}$ of the best particle $\mathrm{g}$ is calculated as the best fitness of all particles. Accordingly, each particle updates its velocity $V_{i d}$ to catch up with the best particle $g$ using equations $(1,2)$. Finally realize image segmentation using optimal $T$ according to the minimize roughness. $T$ bigger than or equal to the minimum gray value and less than or equal to maximum gray value. The proposed image segmentation is described in details in algorithm (1) and illustrated in Fig. 1.

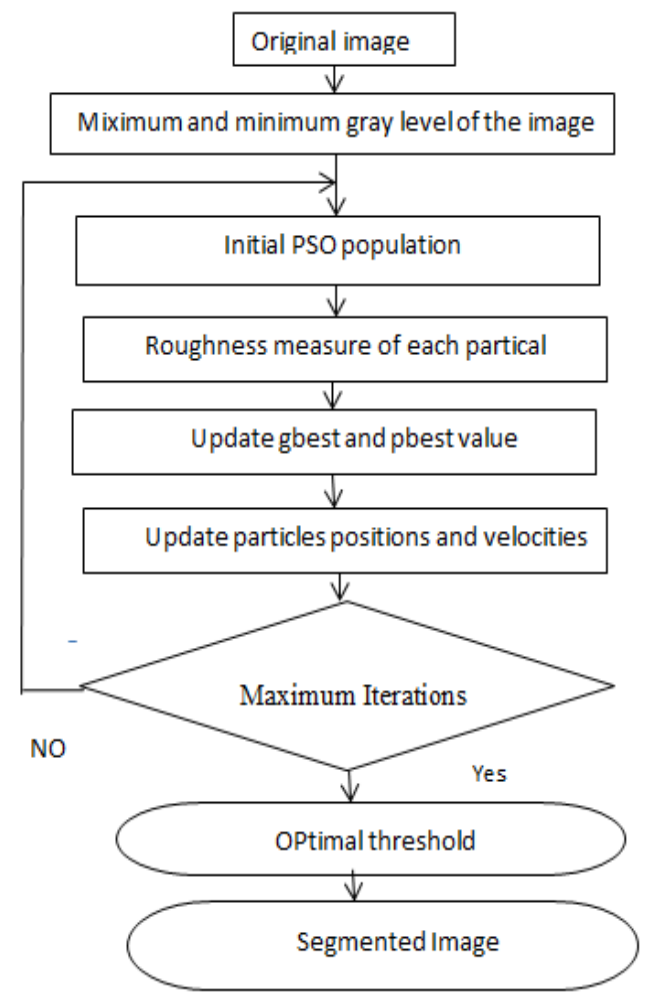

Fig. 1. The proposed flow chart.

\subsection{Image Segmentation Algorithm}

Input: image with maximum gray value $(\max )$ and the minimum value $(\mathrm{min})$

Initialize PSO variables: Number of variable to be optimized, max iteration the maximum number of iteration to consider, $N$ the number of particles and any other problem dependent variables.

1: divide the input image into $M$ blocks

2: Generate initial population of $N$ particles limited in [ $\min : \max ]$

3: FOR $\mathrm{j}=1$ to max iteration

4: FOR i=1 to $N$

5: Calculate object lower and upper using equations $(3,4)$.

6: Calculate background lower and upper using equations $(5,6)$

7. Calculate roughness of object and background using equations (7 and 8).

7: End for

8: Calculate the fitness value of each particle $R M_{T}$ using equation (9).

9: Update the position of gbest and pbest

10: if fitness $\left(X_{i d}\right)<$ fitness pbestid then pbest $_{i}=X_{i d}$

11: else if fitness $\left(X_{i d}\right)<$ fitness gbest $_{d}$ then gbest $_{i}=X_{i d}$

12: End if

13: Update velocity and position of each particle according to equations (1) and (2)

14: Update the value of the weight factor

15: Repeat until the optimum threshold $T$ is found or maximum number of iterations have been reached

16: End for

Output: the segmented image using the optimum threshold $T$ 


\section{Experimental Result}

In this section experiments and results are presented to demonstrate the practice and the performance of the proposed image segmentation algorithm. The proposed algorithm has been implemented in MATLAB platform considering 50 iterations and 25 particles. The algorithm tested on different images with different minimum and maximum gray levels for different block sizes. For the first image with minimum gray level equal 7 and maximum equal 253 which is shown in Fig. 2a, and the segmented images are illustrated in Fig. 2b, Fig. 2c, and Fig. 2 d using block size $2 \times 2,4 \times 4,8 \times 8$ respectively. For the second image with minimum gray level equal 2 and maximum equal 88 which is shown in Fig. 3a, and the segmented images are illustrated in Fig. 3b, Fig. 3 c, and Fig. 3 d using block size $2 \times 2,4 \times 4,8 \times 8$ respectively.

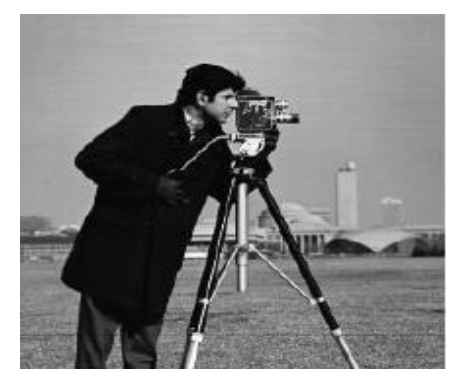

a. Cameraman image

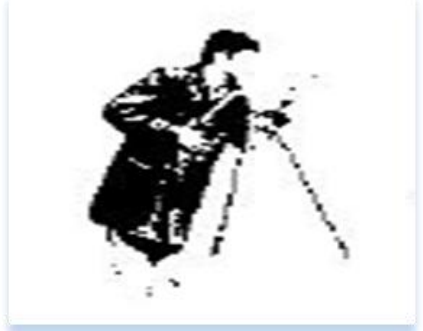

c. Segmented image using $4 \times 4$ block size

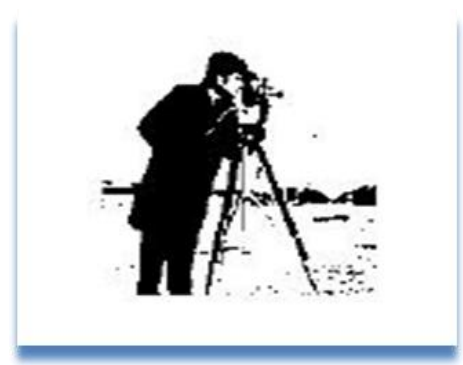

b. Segmented image using $2 \times 2$ block size

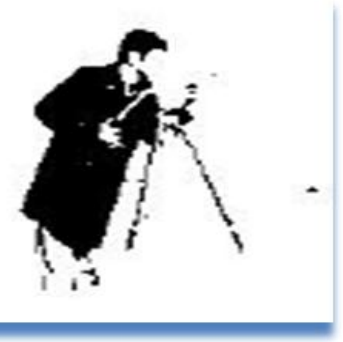

d. Segmented image using $8 \times 8$ block size

Fig.2. Applying the proposed algorithm on cameraman image.
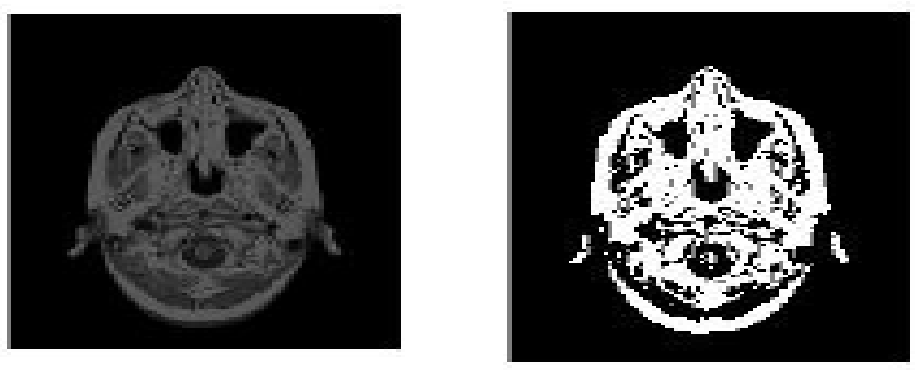

a. MRI image

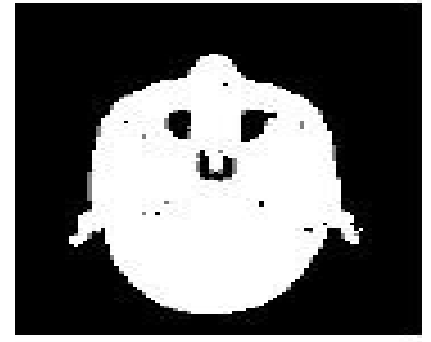

c. Segmented image using $4 \times 4$ block size b. segmented image using $2 \times 2$ block size

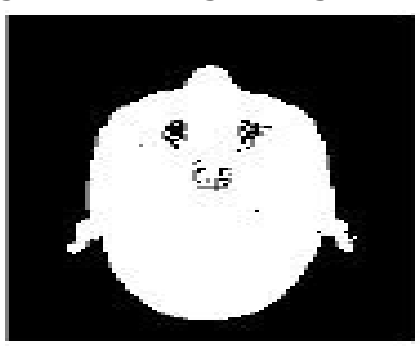

d. segmented image using $8 \times 8$ block size

Fig. 3. Applying the proposed algorithm on MRI image. 
Table 1 illustrates the experimental results. For the first image the minimum roughness measure is equal to 0.4621 and the optimal threshold equal 103 using block size $2 \times 2$. For the second image minimum roughness measure is equal to 0.4897 and the optimal threshold equal 54 also using block size $2 \times 2$. The simulation results indicate that our approach produces good results because the segmented regions are uniform and homogeneous and as displayed in Fig. 4 minimizing block size decreasing the roughness measure.

Table 1. $P S O$ Based Experimental Results

\begin{tabular}{cccc}
\hline \hline block size & $2 \times 2$ & $4 \times 4$ & $8 \times 8$ \\
\hline \multirow{2}{*}{ cameraman image } & $R M=0.4621$ & $R M=0.5001$ & $R M=0.5201$ \\
& $T=103$ & $T=22$ & $T=16$
\end{tabular}

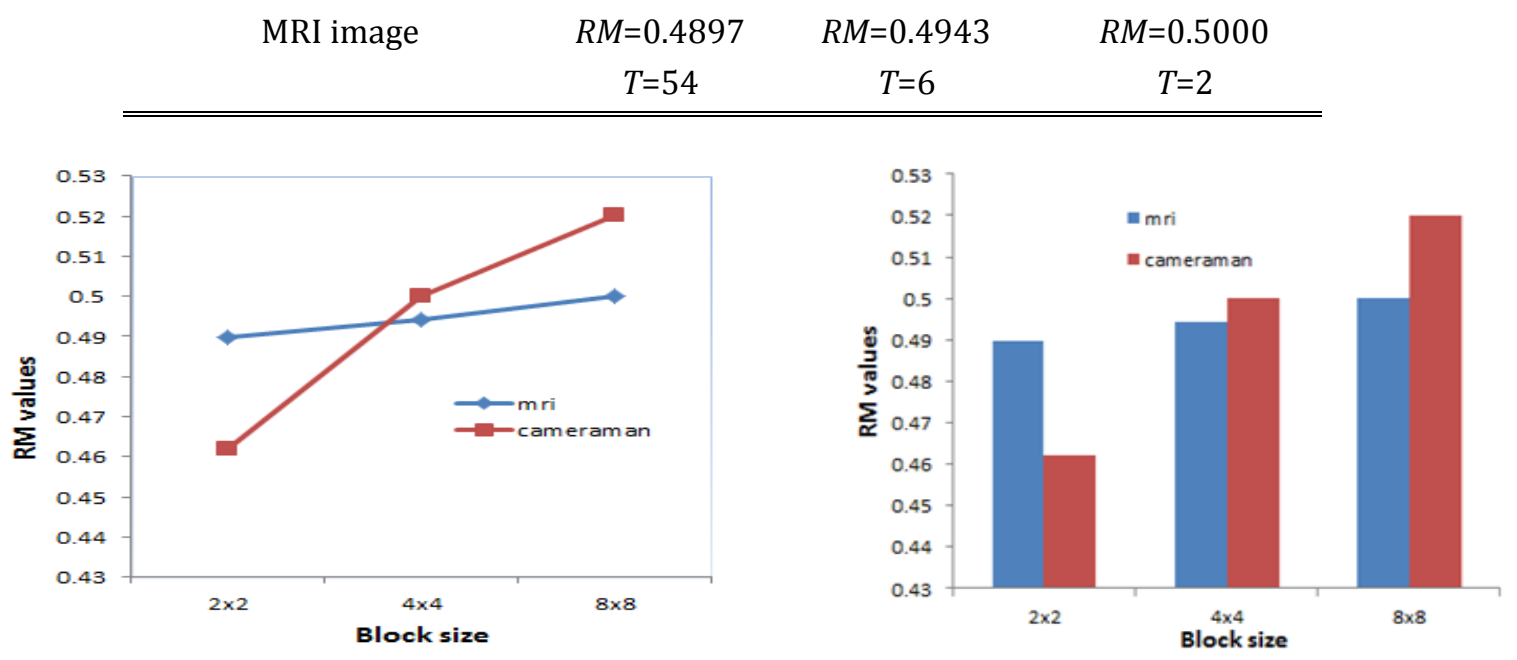

Fig. 4. Display the experimental results.

\section{Comparison with Bat Algorithm}

Bat algorithm is a recent meta heuristic algorithms introduced by Yang in [16], based on echolocation of the bats. In this algorithm, bats detect prey and avoid the obstacles by using the echolocation. Each bat is associated with a velocity $v_{i}(t)$ and a location $y_{i}(t)$, at iteration $t$, in a d-dimensional search or solution space. Among all the bats, there exists a current best solution $y^{*}$ Therefore, the above three rules can be translated into the updating equations for $y_{i}(t)$ and velocities $v_{i}(t)$,

$$
\begin{gathered}
f=f_{\text {min. }}+\left(f_{\text {max }}-f_{\text {min }}\right) \beta \\
v_{i}(t)=v_{i}(t-1)+\left(y_{i}(t-1)-y^{*}\right) f_{i} \\
y_{i}(t)=y_{i}(t-1)+v_{i}(t)
\end{gathered}
$$

where $\beta$ is a random vector generated by a uniform distribution belonging to the closed interval $[0,1]$. The additional control parameters $f_{\min }$ and $f_{\max }$ were set to 0 and 2.0, respectively. The initial values for parameters pulse rate and loudness were set to 0.5. Table 2 illustrates Bat based experimental results.

Table 2. Bat Based Experimental Results

\begin{tabular}{c|c|c|c}
\hline \hline block size & $2 \times 2$ & $4 \times 4$ & $8 \times 8$ \\
\hline \multirow{2}{*}{ cameraman image } & $R M=0.46338$ & $R M=0.50205$ & $R M=0.52997$ \\
& $T=76$ & $T=15$ & $T=18$
\end{tabular}




\begin{tabular}{c|c|c|c}
\multirow{2}{*}{ MRI image } & $R M=0.49022$ & $R M=0.5000$ & $R M=0.54545$ \\
& $T=59$ & $T=7$ & $T=11$ \\
\hline
\end{tabular}

Based on the results which are illustrated in Table 1 and Table 2, the values of mean roughness measure in PSO based proposed algorithm is smaller than in Bat for MRI and cameraman image. Fig. 5 displays the comparison results for MRI image, it can be concluded that the of PSO gives the best segmentation result than Bat.

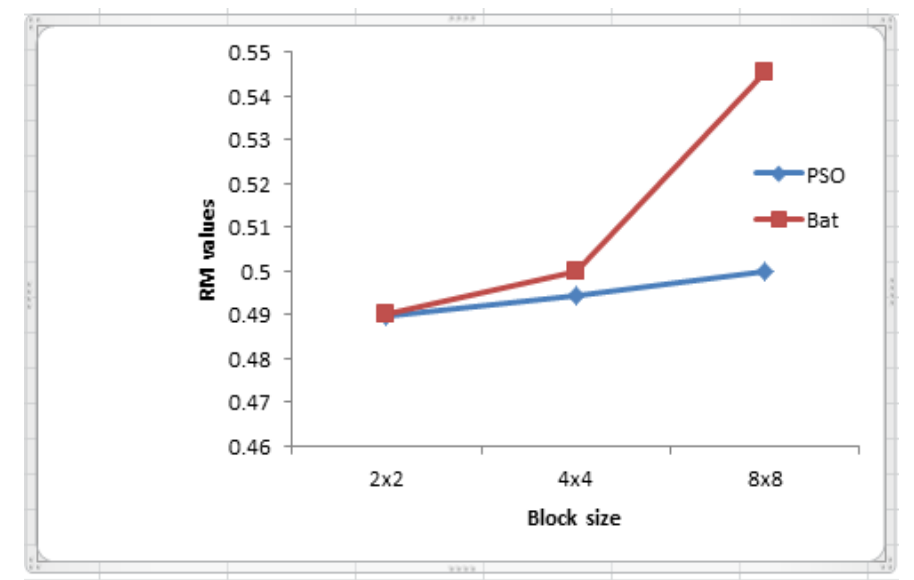

Fig. 5. Display the comparison results.

\section{Conclusion}

In this paper, particle swarm optimization has been merged with rough set to produce a new image segmentation algorithm based on roughness measure. Extracting object from an image is described by minimizing roughness measure using optimal threshold. The optimal threshold is optimized by using PSO together with minimum cross roughness. PSO is widely used due to the strong performance in the optimization. Based on the experimental results as combined PSO with other computational intelligence techniques as rough set the results are more effective and as minimizing block size decreasing the roughness measure thereby providing optimum results for object-background segmentation. PSO based proposed algorithm is observed to be having good performance than Bat.

\section{References}

[1] Anita, T., Rohit, R., \& Yamini, C. (February 2014). Image segmentation based on particle swarm optimization technique. International Journal of Science, Engineering and Technology Research, 3(2).

[2] Behera, H. S. (July 2011). Segmentation and classification using heuristic HRSPSO. International Journal of Soft Computing and Engineering, 1(3).

[3] Amanpreet, K., \& Singh, M. D. (August 2012). An overview of PSO-based approaches in image segmentation. International Journal of Engineering and Technology, 2(8), 1349-1357.

[4] Valentin, O., Erik, C., \& Humberto, S. (2013). A comparison of nature inspired algorithmsfor multi-threshold image segmentation. Expert Systems with Applications, 40, 1213-1219.

[5] Miao, M., Jianhui, L., Min, G., Yi, F., \& Yilong, Y. (2011). SAR image segmentation based on Arti_cial Bee Colony algorithm. Applied Soft Computing, 11, 5205-5214.

[6] Sankar, K. P., Uma, S. B., \& Pabitra, M. (2005). Granular computing, rough entropy andobject extraction. Pattern Recognition Letters, 26, 2509-2517.

[7] Zhang, X., \& Shang, J. (2009). Application of image segmentation algorithm based onparticle swarm optimization and rough entropy standard. Chinese Control andDecision Conference, 2905-2909.

[8] Kennedy, J., \& Eberhart, R. C. (1995). Particle swarm optimization. Proceedings of the IEEE 
International Conference on Neural Networks (pp. 1942-1948).

[9] Singiresu, S. R. (1996). Engineering Optimization Theory and Practice. Wiley Eastern, New Delhi.

[10] Fahd, M., Mohiy, H., Kamel, M., \& Khalid, A. (September 2012). A new image segmentation method based on partical swarm optimaization. The International Arab Journal of Information Tachnology, 9(5), 487-493.

[11] Chih-Chin, L. (January 2006). A novel image segmentation approach based on partical swarm optimaization. IEICE Trans. Fundamentals, 1, 324-328.

[12] Komorowski, J., Pawlak, Z., Polkowski, L., \& Skowron, A. (1999). Roughsets: A Tutorial, Rough Fuzzy Hybridization. Springer-Veralg, 3-98.

[13] Wygralak, M. (1991). On the Power of a Rough Set, 521, Springer-Verlag, 183-192.

[14] Pawlak, Z., Grzymala-Busse, J., Slowinski, R., \& Ziarko, W. (1995). Rough sets. Communications of the ACM, 38(11).

[15] Gaber, A. S., Neveen, I. G., \& Wafaa, A. G. (2008). Object-oriented image segmentation in presence of uncertainty. Advances in Fuzzy Sets and Systems, 3(3), 367-377.

[16] Yang, X. S. (2010). A new metaheuristic bat-inspired algorithm. Studies in Computational Intelligence, $284,65-74$.

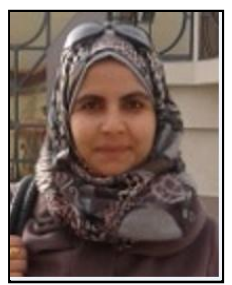

Wafaa Ghonaim received her B.Sc. degree with honors in computer science and pure mathematics from faculty of Science, Al-Azhar University, in 2005. She received her M.Sc. and Ph.D. in computer science from faculty of Science, Al-Azhar University, Cairo, Egypt in 2009, 2013. She is working as a lecture at Faculty of Science, Al-Azhar University. Her current research interest is in the area of computational intelligence based on rough set theory, swarm optimization, image processing, and image retrival. In addition, Dr. Ghonaim is interested in the area of evolutionary computation in cryptanalysis.

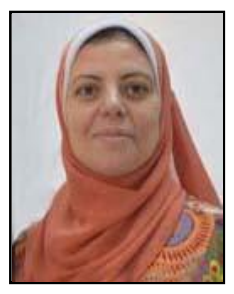

Shereen A. Taie is an associate professor in Computer Science Department, Faculty of Computers and Information, Fayoum University, Egypt and Head of Center of Electronic Courses Production, Fayoum University, Egypt. She held an M.S. in 2006 in Computer Science from Computer and Mathematics Department Faculty of Science, Cairo University, Egypt, and a Ph.D. in 2012 in Computer Science from Computer and Mathematics Department Faculty of Science, Cairo University, Egypt. She was awarded a B.Sc. degree in Computer Science by Faculty of Science, Cairo University in 1996.

She was the head of IT Unit in Faculty of Computers and Information, Fayoum University, Egypt, and Director of Quality Assurance Unit in Faculty of Computers and Information, Fayoum University, Egypt. Also, she was a Staff Member, in Jubail College of Education for Girls, Dammam University, Kingdom of Saudi Arabia, and the Head of Computer and data analysis laboratory, Center for Environmental Hazard Mitigation, Faculty of Science, Cairo University, Egypt.

Dr. Taie is the author of "Online Price Recommendation System for Shopping Used Cell Phones", Research Journal of Applied Sciences, Engineering and Technology, "Challenge of Intelligent Transport System", International Journal Of Modern Engineering Research (IJMER), "A Prototype for Breast Cancer Detection and Development Probability Expert System - Towards a Supportive Tool", The 5th IEEE International Conference on E-Health and Bioengineering - EHB-IEEE, Artificial Intelligent E-learning book (Published by Ministry of Higher Education and Scientific Research), A New Model for Automatic Raster-to- Vector Conversion, International Journal of Engineering and Technology, and "A New Parallel Neural Network System for Automatic Change Detection and Classification of Digital Images", Ain Shams University, Faculty of 
Engineering. She is also a member in Scientific and Technical Committee \& Editorial Review Board on Electrical and Computer Engineering. Finally, she was awarded the first place in the student activities from Dammam University-Kingdom of Saudi Arabia. She was an elite reviewer in Journal of Medical Imaging and Health Informatics (JMIHI). 